\title{
HOMOGENEITY MEASURES FOR MULTIPHASE LEVEL SET SEGMENTATION OF BRAIN MRI
}

\author{
Elsa Angelini ${ }^{1}$, Ting Song ${ }^{2}$, Andrew Laine ${ }^{2}$ \\ ${ }^{1}$ Département Traitement du Signal et des Images, CNRS UMR 5141 LTCI, Ecole Nationale \\ Supérieure des Télécommunications, Paris, France \\ ${ }^{2}$ Department of Biomedical Engineering, Columbia University, New York, NY, USA
}

\begin{abstract}
This paper presents a new homogeneity measure for variational segmentation with multiple level set functions. We propose to modify the quadratic homogeneity measure to trade off the convexity of the function against a faster rate of convergence. We tested in two series of experiments the performance of this new homogeneity force at converging to appropriate partitioning of brain MRI data sets, over a large range of image spatial resolution and image quality, in terms of tissue homogeneity and contrast.

\section{INTRODUCTION}

Although numerous methods to segment brain MRI for extraction of white matter (WM), gray matter (GM) and cerebro-spinal fluid (CSF) have been proposed over the past two decades, little work has been done to evaluate and compare the performance of different segmentation methods on real clinical data sets as well as the performance of a single segmentation method on different clinical data sets.

We previously compared the multiphase level-set segmentation framework of Chan and Vese to four other segmentation methods for segmentation of cortical brain structures [1]. This study lead us to observe very different behaviors of the level-set segmentation method for different brain MRI data sets. In particular, the segmentation method had some difficulty to converge to a satisfactory partitioning of the data when contrast between the GM and the WM was too low. Identified specific weaknesses of the "'best-partitioning"' segmentation method as proposed by Chan and Vese included:(1) sensitivity of the homogeneity measure to image contrast, (2) sensitivity of the homogeneity measure to the gray level range of values, (3) sensitivity of the solution to the initialization configuration.
\end{abstract}

\subsection{Cortical brain segmentation problem statement}

We illustrate in Figure 1 the segmentation problem of extracting cerebral cortical tissues from T1-weighted MRI data on two data sets from two databases, detailed in the Experiments section, with different image quality (with respect to tissue homogeneity and contrast) and spatial resolution. Gray value distribution plots were generated by masking the data with the available manually labeled data.
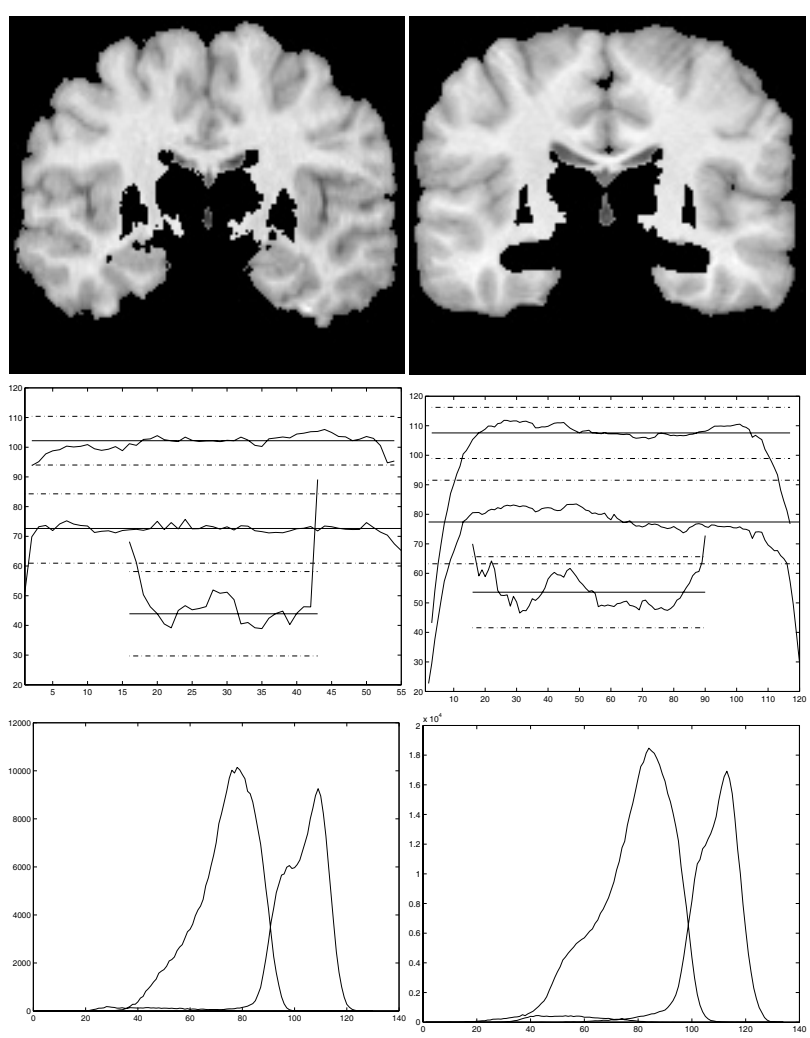

Figure 1: Two brain data sets: from CU (left) and from the IBSR (right). Top row: coronal slices. Middle row: slice-based gray scale average values for GM, WM and CSF, bottom row: histograms of GM, WM and CSF.

These plots illustrate the acceptability of using a grayscale 3D homogeneity measure for separating WM, GM and CSF. They also show the limitations of "simple" direct partitioning approaches such as intensity thresholding (cf. overlap of gray scale distributions of the three tissues in the histogram) or Gaussian statistical modeling of tissue classes (cf. large spectrum of tissue gray values, variation of tissue mean gray values). The CSF class is especially challenging as it is much smaller in terms of sample size and its spectrum is completely overlapped by the tail of the white matter.

\section{SEGMENTATION FRAMEWORK}

\subsection{Variational minimal partitioning}


The segmentation framework used in this study is based on the multiphase formulation of the Mumford and Shah variational framework [2] simplified for piecewise constant data [3] . This approach provides a variational framework for the segmentation of a given image $u_{0}$ defined on $\Omega$ into piecewise constant partitions $\Omega_{i}$ defined by the curve $C$. Given a single closed curve, the underlying "minimal partition problem" is formulated as the minimization of the following functional:

$$
\begin{aligned}
& E=\mu(\text { length }(C))+v(\text { area }(\text { inside } C))+ \\
& \lambda_{1} \int_{\text {inside }(C)}\left|u_{0}-c_{1}\right|^{2} d x+\lambda_{2} \int_{\text {inside }(C)}\left|u_{0}-c_{2}\right|^{2} d x
\end{aligned}
$$

where $\mu \geq 0, v \geq 0, \lambda_{1}, \lambda_{2}>0$ are fixed parameters.

The minimum of the energy is reached by iteratively deforming the curve $C$ and evaluating the $c_{i}$ as the average value of $u_{0}$ inside and outside this curve $C$, splitting the image data into two partitions $\left\{\Omega_{i}\right\}_{i=1,2}$.

This problem was formulated into a level set framework by Chan and Vese in [3] with volume integrals, based on Heaviside and characteristics functions $\chi_{i}$ for each partition $\Omega_{i}$. An alternative was recently proposed by Jehan-Besson et al. who derived a minimization approach for region-based variational segmentation approaches using shape gradients [4].

Extension of the minimal partitioning segmentation framework to multiple objects was proposed by Chan and Vese in [5], defining multiple homogeneous partitions in the image with several curves $\left\{C_{1}, C_{2}, \ldots, C_{i}\right\}$. In the case of two curves $\left(C_{1}, C_{2}\right)$, a segmentation of the image data into four partitions is performed by minimizing the following energy.

$$
\begin{aligned}
& E\left(c_{i, j}, C_{i}\right)=\sum_{i, j=1}^{2} \lambda_{i, j} \int\left|u_{0}-c_{i j}\right|^{2} \chi_{i, j} d \Omega+ \\
& \left.\sum_{i=1}^{2} \mu_{i} \text { length }\left(C_{i}\right)+v_{i} \text { area(inside } C_{i}\right)
\end{aligned}
$$

The level set implementation of the energy minimization framework is detailed in [5].

\subsection{New homogeneity measure}

An intuitive approach to alleviate some of the limitations of the standard approach in providing a robust global minimum to the partitioning problem is to modify the homogeneity measure to a non-strictly convex function, but with still one minimum and sharper slopes towards this minimum. In this context, we propose to use a general $\alpha$-norm, $\alpha \in \mathbb{R}$ with:

$$
\begin{aligned}
& E=\mu(\text { length }(C))+v(\text { area }(\text { inside } C))+ \\
& \lambda_{1} \int_{\text {inside }(C)}\left|u_{0}-c_{1}\right|^{\alpha} d x+\lambda_{2} \int_{\text {inside }(C)}\left|u_{0}-c_{2}\right|^{\alpha} d x
\end{aligned}
$$

The use of the absolute value enforces the symmetry around the optimal homogeneity measure ${ }^{c_{i}}$. A plot of this homogeneity measure and its derivative with respect to $c_{i}$ is provided in Figure 2 for values of $\alpha \in\left[\begin{array}{ll}0 & 0.5\end{array}\right]$.
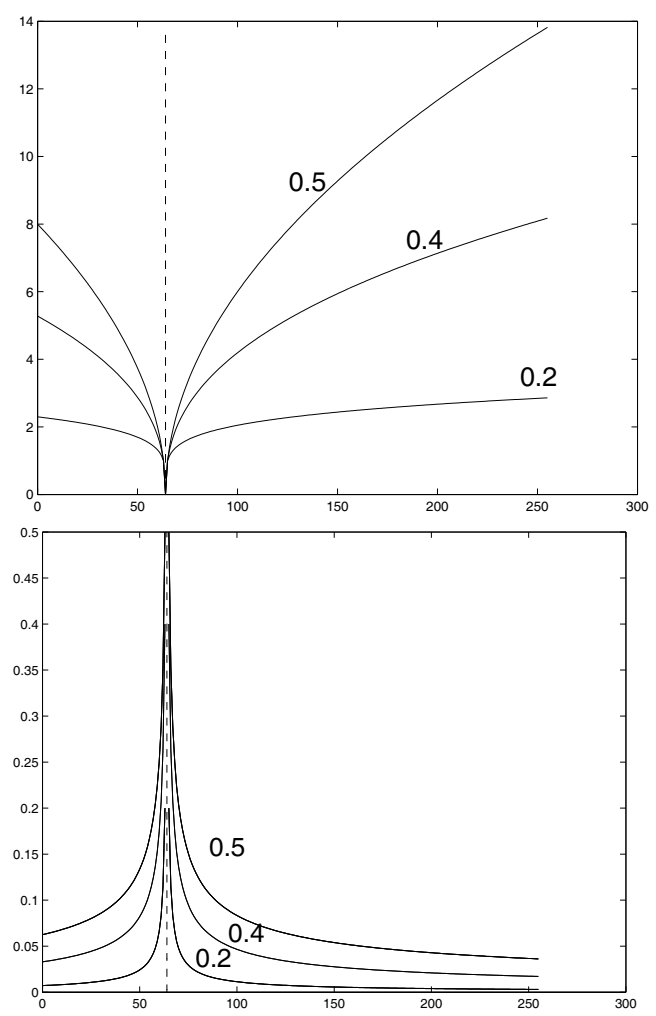

Figure 2: $\alpha$-homogeneity measure and its derivative with respect to $c_{i}$.

We observe in this figure the "wells" shape of the homogeneity function around the homogeneity measure $c_{i}$ with steepest slopes as $\alpha$ increases from 0 to 0.5 .

To derive the Euler-Lagrange equation with respect to the homogeneity measures $c_{i}$ we rewrite the homogeneity terms of the energy function with the help of the characteristics functions $\chi_{i}$ of each region $\Omega_{i}$ (derived from the Heaviside function as defined in [3] ):

$$
\begin{aligned}
E_{\mathrm{hom}} & =\sum_{i} \lambda_{i} \int_{\Omega}\left|u_{0}-c_{i}\right|^{\alpha} \chi_{i} d \Omega= \\
& =\sum_{i} \lambda_{i}\left[\int_{u_{0}>c_{i}}\left(u_{0}-c_{i}\right)^{\alpha} \chi_{i} d \Omega+\int_{u_{0} \leq c_{i}}\left(c_{i}-u_{0}\right)^{\alpha} \chi_{i} d \Omega\right]
\end{aligned}
$$


Derivation of this term with respect to $c_{i}$ leads to the following expression:

$$
\frac{\partial E_{\mathrm{hom}}\left(c_{i}, C\right)}{\partial c_{i}}=\sum_{i} \alpha \lambda_{i} \int_{\Omega} \operatorname{sign}\left(c_{i}-u_{0}\right) \times\left|c_{i}-u_{0}\right|^{\alpha-1} \chi_{i} d \Omega
$$

Minimizing the energy functional therefore corresponds to setting the homogeneity measure $c_{i}$ to the value that equally partitions the area under the image-based derivative function, given a certain geometry of the contour (i.e. $\chi_{i}$ fixed). It can also be seen as the value that splits the distribution of the image voxels in half. The average value, defined as:

$$
c_{i}=\int_{\Omega} u_{0} \chi_{i} d \Omega / \int_{\Omega} \chi_{i} d \Omega
$$

has the property of splitting the distribution of $\left(u_{0}-c_{i}\right)$ in half and therefore cancels out the derivative in Equation (4).

\subsection{Parameters Setting}

Discretization of the minimization problem is described in [3] and [5]. For a 4-phase segmentation with 2 level set functions we have the following set of parameters: $\left\{\lambda_{i, j}, \mu_{i}, v_{i}, \Delta t, \varepsilon\right\}$, where the $\varepsilon$ parameter is used in the definition of the regularized Dirac and Heaviside functions. These parameters need to be carefully scaled for convergence and stability of the algorithm. In particular, the $\varepsilon$ parameter, which controls the area of influence of the Dirac function, is estimated on the level set $\phi$ function (defined for a contour $C$ ) and should be rescaled according to the numerical evolution of the range of values of this function. We updated the value of this parameter at each iteration, setting $\varepsilon^{n}=\max \left(\phi^{n}\right)$ in our implementation and did not use any reinitialization of the level set function.

The range of values of the homogeneity force $\left|u_{0}-c_{i j}\right|^{\alpha}$ depends on the image data values $u_{0}$ as well as the average value of the partition $\Omega_{i j}$. To easily balance this force with the regularizing force, it is desirable to scale this force with respect to the contrast of the image data as follows:

$$
C t=\max \left(u_{0}\right)-\min \left(u_{0}\right), E_{\mathrm{hom}}=\sum_{i} \lambda_{i} \int_{\Omega} \frac{\left|u_{0}-c_{i}\right|^{\alpha}}{C t^{\alpha}} \chi_{i} d \Omega(6)
$$

This normalization provides an homogeneity force with values in the interval [ $\left[\begin{array}{ll}0 & 1\end{array}\right]$. An issue remains though as the scale of this homogeneity force still depends on the average values of the partitions. Indeed, the homogeneity measures from each partition compete with each other, at each image location, to define the overall value added or subtracted to the level set function. This problem has not yet been addressed in our implementation of this segmentation framework.

The time increment parameter $\Delta t$ can be arbitrarily set to any value, when using a semi-implicit discretization scheme for the curvature force, as proposed in [5]. The influence of this parameter on the speed of convergence was directly observed on all the experiments performed on the various data sets.

The regularization parameters were set to 0 for the area terms $v_{i}$ and inversely proportional to the homogeneity parameter for the curvature: $\lambda_{i j}=\lambda, \mu_{i}=\mu=1 / \lambda$.

For the experiments presented in this paper we set $\alpha=0.4, \Delta t=1, \lambda=100$ and obtained convergence in at most 20 iterations.

\subsection{Initialization}

It is desirable to initialize the level set functions with objects from which a distance is simple to compute. It is also desirable to initialize each phase similarly if we don't introduce any a priori information. To fulfill these two requirements, we chose to use two sets of cylinders regularly interleaved over the image data. The dimension of these cylinders is not important, as long as they define 4 phases (i.e they overlap somewhere).

\section{EXPERIMENTAL DATA SETS}

We tested the segmentation on 2 different MRI databases using the same sets of parameters and the same initialization setup:

1. The first database consisted of 10 patients screened with a T1-weighted MRI protocol at the Columbia Presbyterian Medical Center on healthy young volunteers (referred to as the CU database). The MRI data sets were of axial size $(256 \times 256)$ with an in plane resolution of $0.86 \mathrm{~mm}$ and a $3 \mathrm{~mm}$ slice thickness. These data sets have been previously labeled via a labor-intensive manual methods based on histogram thresholds and locally handdrawn regions of anatomical interest.

2. The second data set was obtained on the Internet Brain Segmentation Repository from the Center for Morphometric Analysis at the Massachusetts General Hospital (IBSR: http://www.cma.mgh.harvard.edu/ibsr) which provides manually-guided expert segmentation results along with MRI brain image data. We used the database of 18 patients with in plane resolution ranging from $0.84 \mathrm{~mm}$ to $1 \mathrm{~mm}$ (matrix size $256 \times 267$ ) and $1.5 \mathrm{~mm}$ slice thickness. These data sets have been pre-processed for bias-field correction.

All MRI volumes were pre-processed to remove all noncortical brain tissue by using the manually labeled data 
sets as binary masks.

\section{RESULTS}

The overlap and difference between our segmentation and manual labeling was measured for true positive $(T P)$, false positive $(F P)$ and false negative $(F N)$ volume fractions $(V F)$ of the true delineated object volume. Our previous experiments with the multiphase level set framework using quadratic homogeneity measures on the CU database was presented in [6] and [1]. It required adhoc parameter settings which depended on the data volume dimensions and scaling of the homogeneity force with respect to the average intensities of each phase. Quantitative error measurements reported average errors in the following range for [GM WM CSF]: $\mathrm{FN}=[7 \% 6 \%$

$31 \%] ; \mathrm{FP}=\left[\begin{array}{llll}6 \% & 8 \% & 5 \%\end{array}\right]$ and $\mathrm{TP}=\left[\begin{array}{llll}93 \% & 94 \% & 68 \%\end{array}\right]$ We reproduced similar results with a much simpler algorithmic setup in the present study. With the previous set up, we could not achieve satisfactory segmentation of the entire IBSR database. With the proposed homogeneity measure and parameter setting, we were able to segment the 18 cases included in the database. An illustration of the segmentation quality is provided in Figure 3. Average error measurements were for [GM WM CSF]: $\mathrm{FN}=\left[\begin{array}{lll}24 \% & 24 \% & 8 \%\end{array}\right] \mathrm{FP}=[10 \% \quad 8 \% \mathrm{NA}]$ and $\mathrm{TP}=[74 \%$ $75 \% 92 \%]$. There are still remaining issues with the automated segmentation of this data set and the comparison to the manual labeling as some strong cortical CSF signal was manually labeled as GM. This is a well known limitation for using this database for segmentation validation, and this is why we cannot report a meaningful FP volume fraction of CSF.

\section{DISCUSSION AND CONCLUSION}

In this paper we proposed a modification of the homogeneity metric used in a multi-phase level set segmentation framework for the segmentation of cortical brain structures. The segmentation method was able to handle multiple MRI data sets without any a priori information and with a common setup (i.e. without ad hoc parameterization). To put our work in perspective, we identified two recent papers on segmentation of the IBSR data set: In a recent paper [7], several competitive level set functions were used to segment the brain hemispheres and the cerebellum, and 4 internal structures. Although we cannot compare our results as the cortical cerebral tissues were not segmented, the authors acknowledged in their discussion the difficulty of segmenting the IBSR database because of strong intensity variations from one dataset to another and even inside a single IBSR dataset. In [8], Shen et al. compared the performance of different fuzzy C-means classification algorithms for the segmentation of cortical brain structures. The authors tested the algorithms on 1 IBSR data set, reporting oversegmentation of the three tissues: CSF (39\%), WM (13\%) and GM $(21 \%)$ while under-segmentation and incorrect segmentation statistics were below 3\%. These results compare well with ours for the order of magnitude of errors obtained when comparing to the manual labels, with best accuracy achieved for WM. We also recall that manual labeling is only used as a method of reference, and does not provide a real "ground truth" to the segmentation problem. In that context, Kikinis et al. reported in [9] a variation in volumetric measurements between manual observers in the order of $15 \%$ for WM, GM and CSF.

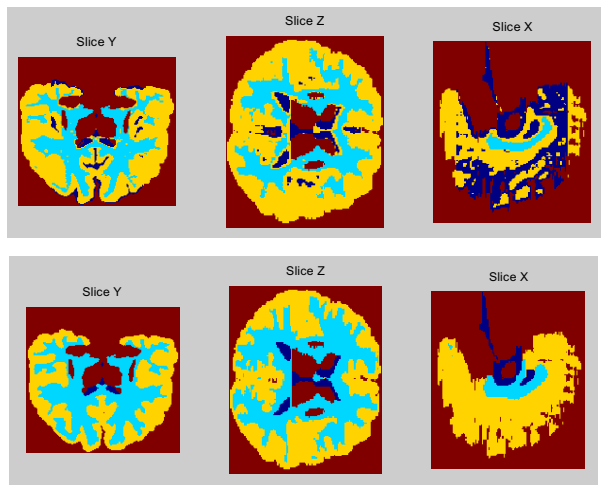

Figure 3: Segmentation the IBSR data into 4 phases. Top row: level set, bottom row: manual labels. Future work will include the extension of the segmentation framework for additional partitioning, to be able to handle the presence of tumoral pathologies in the MRI data. .We are also investigating further modifications of the homogeneity measure to remove its dependency upon the average intensities of each partition.

\section{REFERENCES}

[1] T. Song, E. D. Angelini, B. D. Mensh, and A. Laine, "Comparison study of clinical 3D MRI brain segmentation evaluation," Annual International Conference IEEE Engineering in Medicine and Biology Society (EMBS), San Francisco, CA, USA, pp. 1671-1674, 2004.

[2] D. Mumford and J. Shah, "Optimal approximation by piecewise smooth functions and associated variational problems," Communications on Pure and Applied Mathematics, vol. 42, No., pp. 577-685, 1989

[3] T. F. Chan and L. A. Vese, "Active contours without edges," IEEE Transactions on Image Processing, vol. 10, No. 2, pp. 266 - 277, 2001.

[4] S. Jehan-Besson, M. Barlaud, and G. Aubert, "DREAM2S: Deformable Region driven by an Euclidian Accurate Minimization Method for image and video segmentation," International Journal of Computer Vision, vol. 53, No. 1, pp. 45-70, 2003.

[5] L. A. Vese and T. F. Chan, "A multiphase level set framework for image segmentation using the Mumford and Shah model," International Journal of Computer Vision, vol. 50, No. 3, pp. 271-293, 2002.

[6] E. D. Angelini, T. Song, B. D. Mensh, and A. Laine, "Multi-phase three-dimensional level set segmentation of brain MRI," International Conference on Medical Image Computing and Computer-Assisted Intervention (MICCAI), Saint-Malo, France, pp. 318-326, 2004.

[7] C. Ciofolo and C. Barillot, "Brain segmentation with competitive level sets and fuzzy control," Information Processing in Medical Imaging (IPMI), Glenwood Springs, CO, USA2005.

[8] S. Shen, W. Sandham, M. Granat, and A. Sterr, "MRI fuzzy segmentation of brain tissue using neighborhood attraction with neural network optimization," IEEE Transactions on Information Technology in Biomedicine, vol. 9, No. 3, pp. 459-467, 2005.

[9] R. Kikinis, M. E. Shenton, G. Gerig, J. Martin, M. Anderson, D. Metcalf, C. R. Guttmann, R. W. McCarley, W. Lorensen, H. Cline, and F. A. Jolesz, "Routine quantitative analysis of brain and cerebrospinal fluid spaces with MR imaging," Journal of Magnetic Resonance Imaging, vol. 2, No. 6, pp. 619-629, 1992. 